\title{
Designing, Proposed Synthesis and Docking Analysis of Novel Sulfonamide Derivatives as Antimicrobial Agents
}

\author{
Ajeet ${ }^{*}$, Arvind Kumar \\ Department of Pharmaceutical Chemistry and Drug Design, S. D. College of Pharmacy and Vocational Studies, Muzaffarnagar, India \\ *Corresponding author: ajeet_pharma111@rediffmail.com
}

Received January 27, 2014; Revised April 02, 2014; Accepted April 03, 2014

\begin{abstract}
Substituted N-acetyl-4-amino-benzenesulfonamide derivatives were designed using ChemDraw Ultra 7.0 and energy minimization of derivatives was achieved with Chem3D Pro of ChemOffice suit, keeping in view the structural requirements of pharmacophore. Their proposed synthesis along with in-silico study (docking analysis) in favor of antimicrobial activity has been shown in this work with the possible mechanism of reaction. Docking studies were carried out to study the binding properties of drugs with molecular targets with the help of AutoDock Vina (Python-Prescription 0.8). Titled compounds (CS, DPS, SRS and TS) exhibited good binding properties with molecular target pseudomonas aeruginosa exotoxin A in Lamarckian genetic algorithm based flexible docking studies.
\end{abstract}

Keywords: sulfonamide derivatives, proposed synthesis, antimicrobial, molecular docking

Cite This Article: Ajeet, and Arvind Kumar, "Designing, Proposed Synthesis and Docking Analysis of Novel Sulfonamide Derivatives as Antimicrobial Agents.” American Journal of Pharmacological Sciences, vol. 2, no. 2 (2014): 37-41. doi: 10.12691/ajps-2-2-2.

\section{Introduction}

With serious mortality and morbidity results, drug resistance against bacteria have emerges with public health problem all over the world. The case of penicillin resistance worldwide could be considered as one of the example. Additionally, multi-drug resistance has created another problem to work with. This type of problem could be observed in Europe, Asia and America with vancomycin resistance [1-8]. The above mentioned problems and so many others like these promoted us to contribute hands a little towards solving the problems by proposing synthesis and screening a series of sulfonamide derivatives.

Mode of action of sulfonamide drugs observed so far is inhibition of carbonic anhydrase against a wide range of bacteria. The substituted ring of benzenesulfonamide containing $-\mathrm{SO}_{2} \mathrm{NH}_{2}$ groups act by binding or coordination of the $-\mathrm{SO}_{2} \mathrm{NH}^{-}$anion to the $\mathrm{Zn}^{2+}$ of the enzyme, mimicking the bicarbonate anion in the transition state [9]. The mode of action of antimetabolite sulfa drugs is the inhibition of dihydropteroate synthetase, which catalyzes an enzyme in the biosynthesis of tetrahydrofolate and then nucleotides [10].

In the same way, we have screened (in-silico screening) the designed compounds against Pseudomonas aeruginosa exotoxin A by the means of docking studies.

The Pseudomonas exotoxin A is an exotoxin produced by Pseudomonas aeruginosa. It inhibits elongation factor2. It does so by ADP-ribosylation of EF2 (eukaryotic elongation factor-2). This then causes the elongation of polypeptides to cease.

\section{Material and Methods}

The chemical structures of the derivatives were drawn using ChemDraw Ultra 7.0 and energy minimization of derivatives was achieved with Chem3D Pro of ChemOffice suit for taking energy of each molecule up to its lowest energy state (highest stability).

\subsection{Proposed Synthesis of Substituted Acyl Chlorides}

Substituted acid (0.1 mol) and thionyl chloride (0.4 mol) should place in a $250 \mathrm{ml}$ flask equipped with a magnetic stirrer bar and a condenser with a drying tube. The reaction mixture should stir and heated in a $70^{\circ} \mathrm{C}$ oil bath. After 0.5 hours, the reaction mixture would allow cooling at room temperature with opened flask; this facilitates the evaporation of remaining thionyl chloride and lefts acyl chloride in the flask [11,12].

\subsection{Proposed Synthesis of Substituted N- acetyl-4-amino-benzenesulfonamide}

For the synthesis of an appropriate amide, the substituted acyl chloride $(0.009 \mathrm{~mol})$ of an individual acid dissolved in $20 \mathrm{ml}$. of dry acetone would added drop wise to a stirred solution of aromatic aminosulfonamide $(0.0092 \mathrm{~mol})$ and pyridine $(0.0091 \mathrm{~mol})$ in $50 \mathrm{ml}$. of dry acetone. After addition, the reaction mixture would stir for about 12 hour at room temperature and then the solvent should evaporate under reduced pressure. The residue should then dissolve in $100 \mathrm{ml}$. ethyl acetate and the 
organic phase washed three times with $20 \mathrm{ml}$. of distilled water. Then $10 \% \mathrm{HCl}$ solution was added until $\mathrm{pH} 1$ was reached, and the organic phase was separated from the aqueous phase and washed three times with brine. The aqueous solutions should combine and extracted with ethyl acetate. The ethyl acetate extracts were combined, dried over $\mathrm{MgSO}_{4}$, filtered and evaporated under reduced pressure [11,12].

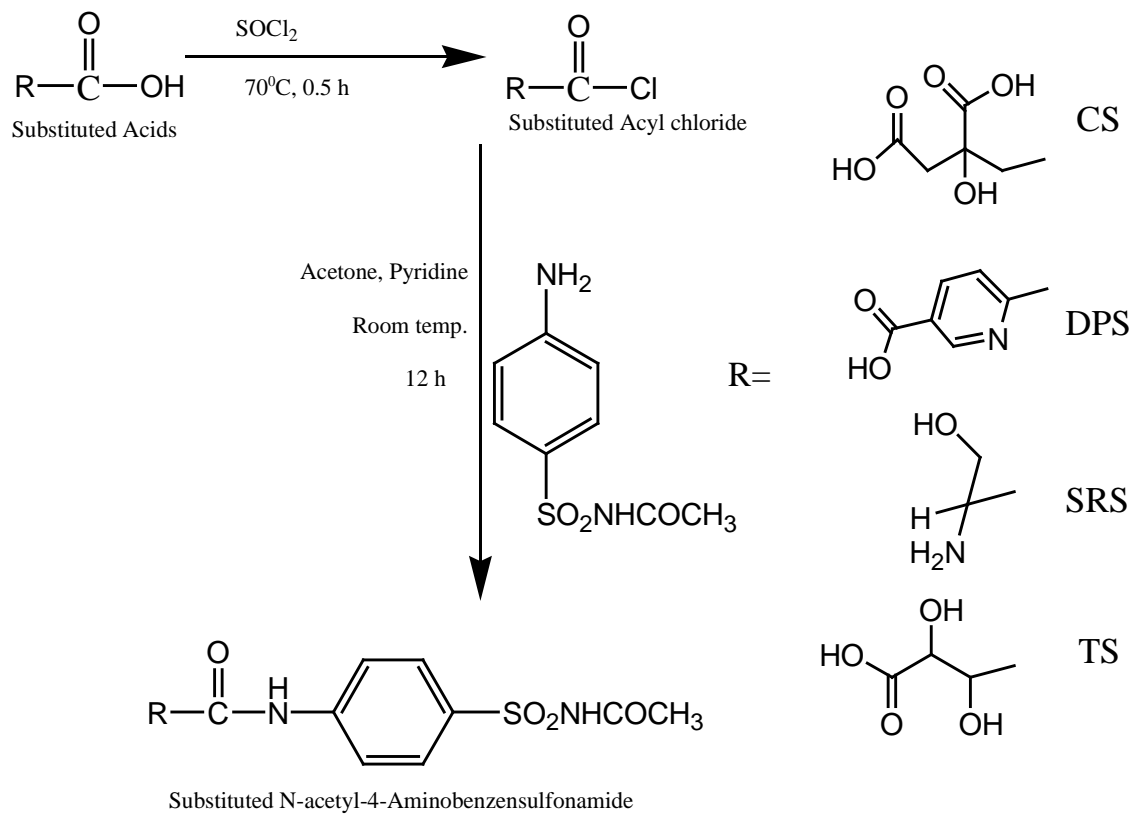

Scheme 1. Proposed synthetic scheme of substituted N-acetyl-4-amino-benzenesulfonamide from substituted acids (CS, DPS, SRS, TS)

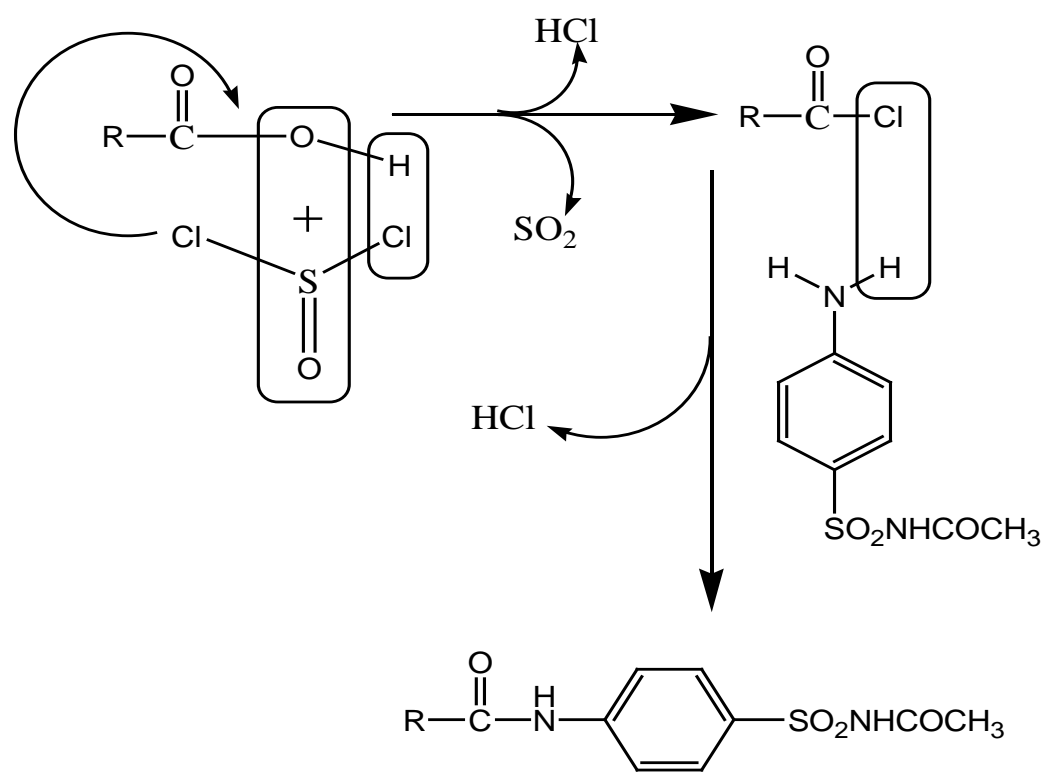

Mechanism 1. Proposed mechanism of reaction

\subsection{In-silico Studies}

\subsubsection{Docking Studies}

\subsubsection{Docking}

Molecular docking techniques are used in modern drug design to help understand drug-receptor interaction. It has been shown in the literature that these computational procedures can strongly support and help the design of new, more potent drugs by revealing the mechanism of drug-receptor interaction. Rational drug design helps to facilitate and speedup the drug designing process, which involves variety of methods to identify novel compound, out of them one method is the docking of the drug molecule with the receptor. The therapeutic action of the clinical drug will be effective when the biochemical pathway of the enzyme can be exploited. Docking procedures allows virtually screening a data-base of compounds and predict the strongest binder based on various scoring functions [13-18].

\subsubsection{Receptor}

Pseudomonas aeruginosa exotoxin A.

\subsubsection{Docking Tool}

Here docking has been performed with AutoDock docking software. It is virtual screening software for computational drug discovery that can be used to screen libraries of compounds against potential drug targets. It enables medicinal chemists to run virtual screening form 
any platform and helps users in every steps of this process from data preparation to job submission and analysis of the results [13-18].

For performing docking, receptor has been downloaded from NCBI website with PDB ID 1IKQ (Pseudomonas aeruginosa exotoxin $\mathrm{A}$ ), then designed ligand has been docked with protein (receptor) with AutoDock software having its default settings.

\section{Results and Discussion}

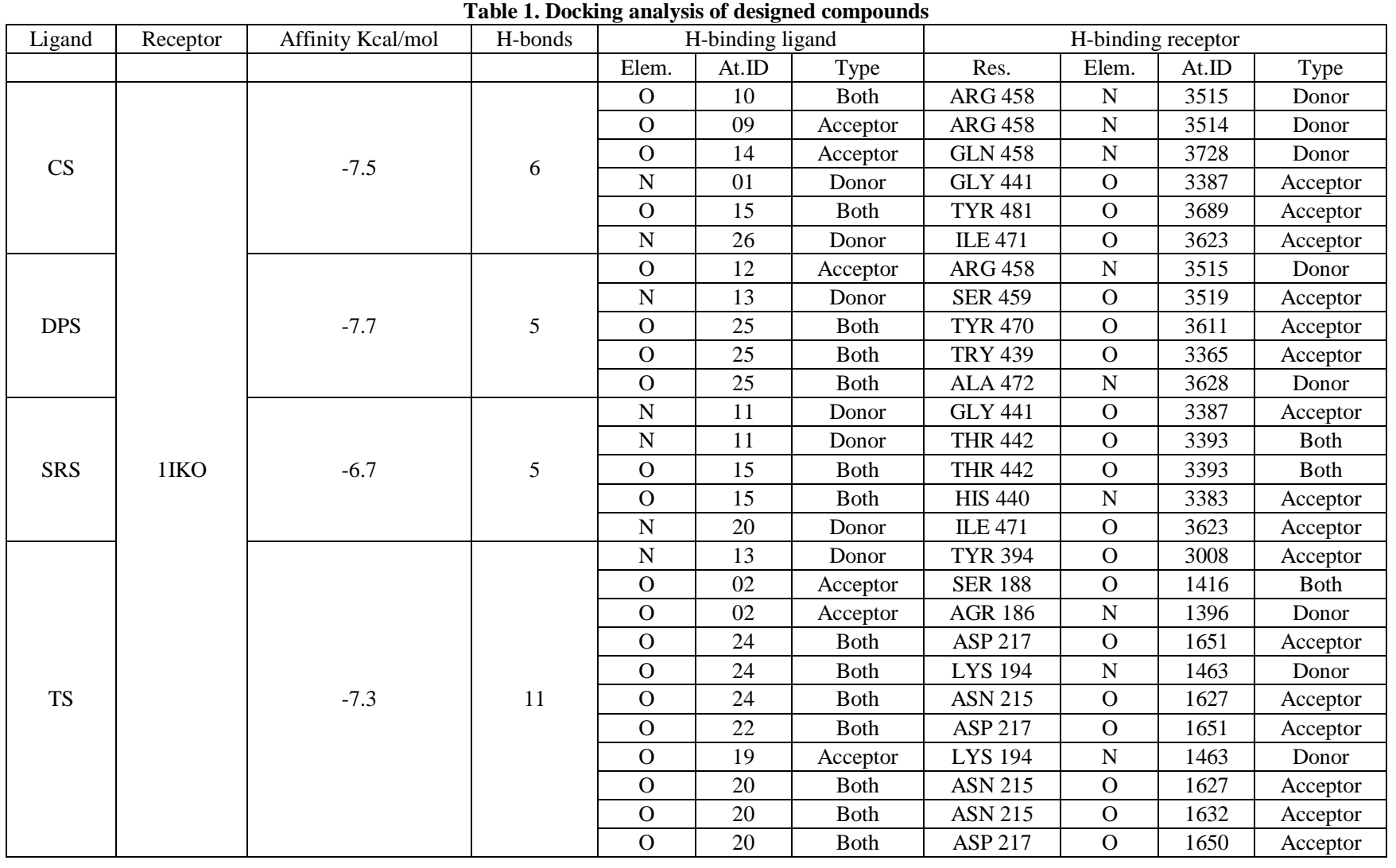

a.

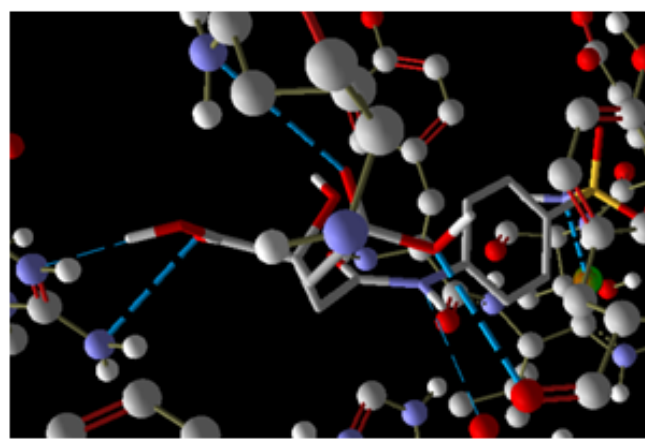

c.

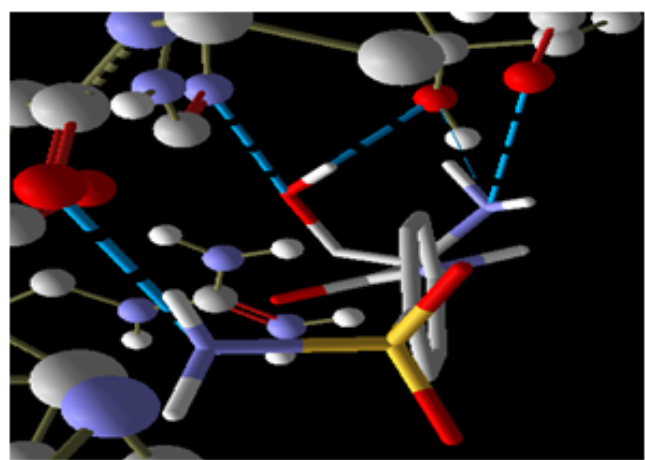

\subsection{Docking Study of N-acetyl-4-amino- benzenesulfonamide Derivatives and Standard Drug Taken Norfloxacin (CID_4539)}

Docking study of protein was performed with the designed inhibitors and standard drug taken is given in Table 1 \& Table 2 and number of hydrogen bonds \& binding pattern such as element, type of bond, atom number and residue at binding site were evaluated.

Table 1. Docking analysis of designed compounds 
On docking analysis, designed compound TS have been found to be strongly docked with the protein 1IKQ in order to study its inhibition activity. When it is docked with the protein PDB ID- 1IKQ, it forms 11 hydrogen bonds with binding affinity of $-7.3 \mathrm{Kcal} / \mathrm{mol}$. On residue study, the amino acids TYR 394, SER 188, ARG 186,
ASP 217, LYS 194 and ASN 215 were found to be significant. While other novel designed molecules CS, DPS and SRS shows moderate binding pattern with -7.5 $\mathrm{Kcal} / \mathrm{mol},-7.7 \mathrm{Kcal} / \mathrm{mol},-6.7 \mathrm{Kcal} / \mathrm{mol}$ and $6,5,5$ hydrogen bonds respectively. Their docking images have been shown in Figure 1.

Table 2. Docking analysis of Norfloxacin (CID_4539)

\begin{tabular}{|c|c|c|c|c|c|c|c|c|c|c|}
\hline \multirow[t]{2}{*}{ Ligand } & \multirow[t]{2}{*}{ Receptor } & \multirow[t]{2}{*}{$\begin{array}{c}\text { Affinity } \\
\text { Kcal/mol }\end{array}$} & \multirow[t]{2}{*}{$\begin{array}{c}\mathrm{H}- \\
\text { bonds }\end{array}$} & \multicolumn{3}{|c|}{ H- Binding Ligand } & \multicolumn{4}{|c|}{ H- Binding Receptor } \\
\hline & & & & Elem. & At. ID. & Type & Res. & Elem. & At.ID. & Type \\
\hline \multirow{4}{*}{ CID_4539 } & \multirow{4}{*}{$1 \mathrm{IKQ}$} & \multirow{4}{*}{-8.1} & \multirow{4}{*}{4} & $\mathrm{O}$ & 23 & Both & GLN 212 & $\mathrm{O}$ & 1604 & Acceptor \\
\hline & & & & $\mathrm{O}$ & 23 & Both & GLU 399 & $\mathrm{O}$ & 3048 & Acceptor \\
\hline & & & & $\mathrm{O}$ & 22 & Acceptor & TYR 206 & $\mathrm{O}$ & 1554 & Both \\
\hline & & & & $\mathrm{O}$ & 22 & Acceptor & GLN 212 & $\mathrm{~N}$ & 1605 & Donor \\
\hline
\end{tabular}

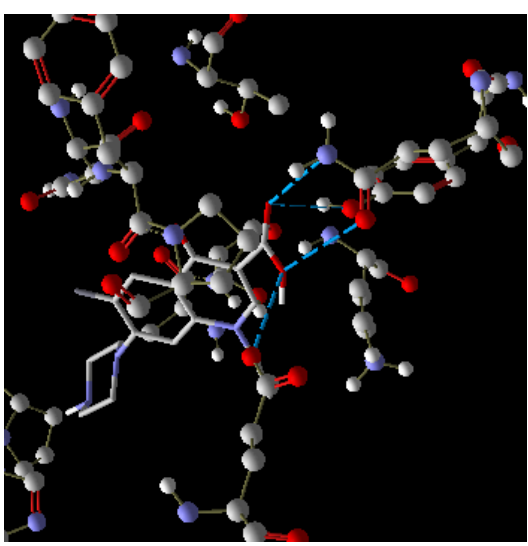

Figure 2. Docked photographs of Norfloxacin (CID_4539) with protein $1 \mathrm{IKQ}$

On docking analysis, the standard drug CID_4539 has been found to be strongly docked with the protein 1IKQ in order to study its inhibition activity. When it is docked with the protein PDB ID- 1IKQ, it forms 4 hydrogen bonds with binding affinity of $-8.1 \mathrm{Kcal} / \mathrm{mol}$. On residue study, the amino acids TYR 206, GLN 212, GLU 399, TYR 206 and GLN 212 were found to be significant. Its docking image has been shown in Figure 2.

\subsection{Comparison of Docking Results of Novel Designed Molecules with the Standard Drug CID_4539}

On docking analysis, the docked poses of DPS, CS and SRS do not superimpose with standard drug molecule which can be clearly seen in Figure 3, but the docking analysis shows that they are nicely docked with protein rather in the catalytic domain, it means it binds in the allosteric site and there is possibility of making it antimicrobial.

On docking analysis of TS, it is clearly observed that this is only the molecule out of four which intercalate with the binding domain of the standard drug taken. (see Figure 3).

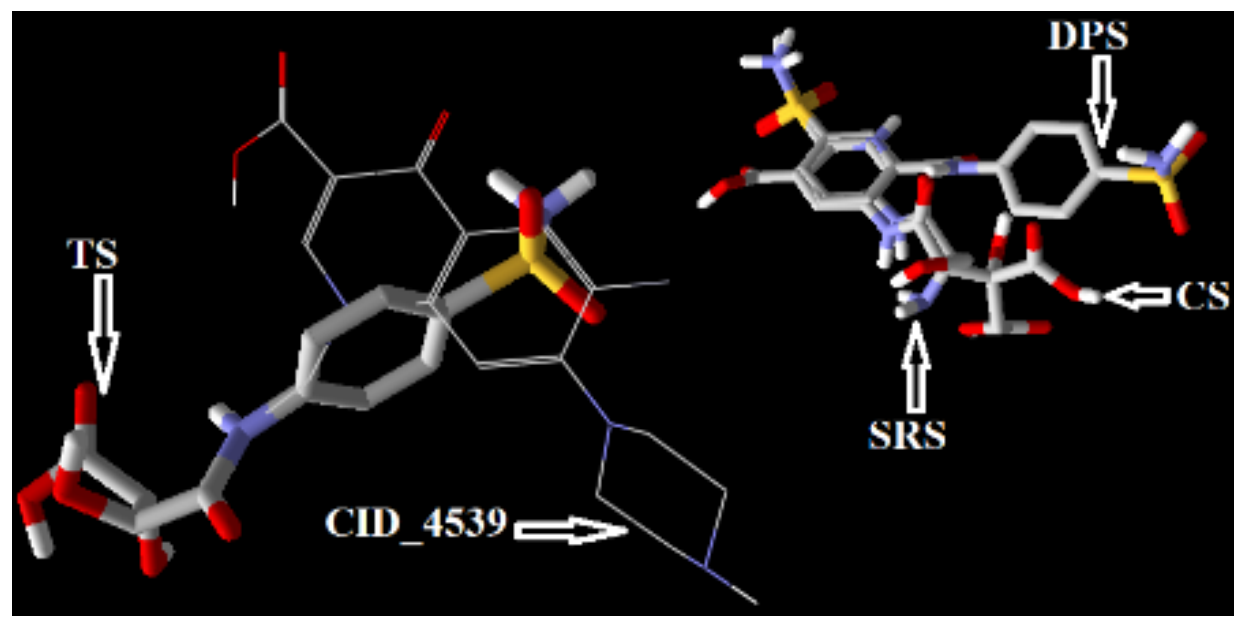

Figure 3. Comparative docking poses of novel designed molecules (showing with stick model) along with standard drug CID_4539 (showing with wireframe model)

\section{Conclusion}

In the present work, a synthetic procedure has been proposed for the synthesis of novel sulfonamide derivatives based on previous works keeping in view the structural requirement of the compound with antimicrobial activity. For, targeting the above problem we designed compounds and evaluated them with docking studies.
In order to obtain substituted acyl chlorides, substituted acids could be treated with thionyl chloride and then substituted acyl chloride could be treated with aminosulfonamides in the presence of pyridine for obtaining the substituted N-acetyl-4-amino-benzenesulfonamides.

The docking result of standard drug taken CID_4539 (Norfloxacin) correlates well with the performance of compound TS in docking study. Hence, it could be concluded that molecular target responsible for the antimicrobial activity of substituted N-acetyl-4-amino- 
benzenesulfonamides may be pseudomonas aeruginosa exotoxin A. Although a proper synthesis, their analytical characterization and systemic biochemical study of novel designed molecules is necessary to confirm the findings.

On comparing the chemical structure of novel designed $\mathrm{N}$-acetyl-4-amino-benzenesulfonamides derivatives with chemical structure of Norfloxacin (1-ethyl-6-fluoro-4-oxo7-piperazin-1-yl-1H-quinoline-3-carboxylic acid), it is concluded that a phenyl ring and a free carboxylic functionality are essential pharmacophoric requirements in designing a pseudomonas aeruginosa exotoxin A inhibitor.

\section{References}

[1] Jones M.E., Blosser-Middleton R.S., Thornsberry C., Karlowsky J.A., Sahm D.F., "The activity of levofloxacin and other antimicrobials against clinical isolates of Streptococcus pneumoniae collected worldwide during 1999-2002", Diagn. Microbiol. Infect. Dis., 47, 579-586, 2003.

[2] Fouda S.I., Kadry A.A., Shibl A.M., (2004) "Beta-lactam and macrolide resistance and serotype distribution among Streptococcus pneumoniae isolates from Saudi Arabia”, $J$. Chemother., 16, 517-523, 2004.

[3] Jenkins S.G., Farrell D.J., Patel M., Lavin B.S., "Trends in antibacterial resistance among Streptococcus pneumonia isolated in the USA, 2000-2003: PROTEK US years 1-3”, J. Infect., 51, 355-363, 2005.

[4] Krcméry V., Sefton A., "Vancomycin resistance in Gram-positive bacteria other than Enterococcus spp.”, Int. J. Antimicrob. Agents., 14, 99-105, 2000.

[5] Kaye K.S., Engemann J.J., Fraimow H.S., Abrutyn E., "Pathogens resistant to antimicrobial agents:epidemiology, molecular mechanisms, and clinical management”, Infect. Dis. Clin. North. Am., 18, 467-511, 2004.

[6] Cuellar-Rodríguez J., et. al. "Vancomycin-resistant enterococci, México City”, Emerg. Infect. Dis., 13, 798-799, 2007.
[7] Murthy A., De Angelis G., Pittet D., Schrenzel J., Uckay I., Harbarth S., "Cost-effectiveness of universal MRSA screening on admission to surgery", Clin. Microbiol. Infect., 16, 1747-1753, 2010.

[8] Thakur A., Thakur M., Supuran, Khadikar P.V., "QSAR study on inhibition of E. Coli by sulfonamides", ARKIVOC, xiv, 593-598, 2006.

[9] Hong Y.L., Hossler P.A., Calhoun D.H., Meshnick S.R. "Inhibition of recombinant Pneumocystis carinii dihydropteroate synthetase by sulfa drugs”, Antimicrob. Agents. Chemother., 39, 1756-1763, 1995.

[10] Furniss B.S., Hannaford A.J., Smith P.W.G., Tatchell A.R., Vogel's Textook of Practical Organic Chemistry; Prentice Hall, New York, 1989.

[11] Naama H., Meir B., Bogdan W., Richard H.F., Boris Y., "Syntheses and evaluation of anticonvulsant profile and teratogenicity of novel amide derivatives of branched aliphatic carboxylic acids with 4-aminobenzensulfonamide”, J. Med. Chem., 53, 4177-4186, 2010.

[12] Ajeet, "Chlorambucil derivatives as antineoplastic agent: in-silico designing and docking”, Int. J. Cur. Pharm. Res., 3(4), 81-84, 2011.

[13] Ajeet, "Trans-disciplinary receptor binding of acyclovir to human phenylalanine hydroxylase: docking approach", Int. J. Pharm. Pharm. Sci., 4(suppl 3), 182-184, 2012.

[14] Ajeet, "In-silico designing and characterization of Amiloride derivatives as ion channel modulator”, Med. Chem. Res., 22(2), 1004-1010, 2013.

[15] Ajeet, Kumar P., Tripathi L., "Virtual Screening Tool Based Designing and Evaluation of Novel Sulfonamide Derivatives as Anticonvulsant Agent - A Pharmacophoric Approach”, Int. J. Pharm. Phytopharmacol. Res., 2(3), 202-208, 2012.

[16] Ajeet, Tripathi L., Kumar P., "Designing of Novel 6(H)-1,3,4Thiadiazine Derivatives as MMP12 Inhibitors: A MLR and Docking Approach", American Journal of Pharmacological Sciences,1(2), 29-34, 2013.

[17] Ajeet, Kumar A., "Designing of Hybrid form of Benzothiazolequinazoline as GABA-A Inhibitor with Anticonvulsant Profile: An in-silico Approach", American Journal of Pharmacological Sciences, 1(6), 116-120, 2013. 